\title{
INVESTIGATING A REPORTED SCARLET FEVER OUTBREAK
}

hildren at a day care centre were involved in a suspected outbreak of scarlet fever investigated by the Illawarra Public Health Unit in June this year. The Shellharbour Council notified the Illawarra PHU on June 18 of a suspected outbreak of scarlet fever in a day care centre at Albion Park Rail. The director of the centre had contacted the council reporting four 'confirmed' cases and three 'suspected' cases of scarlet fever. The basis of this report for the centre director was verbal reports from the mothers of the children on the basis of clinical diagnoses by their doctors. The director responded by placing a notice to the parents on the front door of the centre, warning them of a scarlet fever outbreak.

The day care centre is a community child care centre, whose 72 attending children are between the ages of one and five (inclusive). Up to 40 children attend the centre each day. Staff at the centre comprises six full-time, two casual relief and two part-time workers.

Each day the children are put into two groups, one with about 15 children aged one to three and the other with about 25 children aged three to five. These two groups use separate rooms for part of the day, although there are times in the morning and afternoon when the children are together.

The index case was a four-year-old girl from Albion Park who became ill on June 2 with a sudden onset of fever and sore throat. The next day a rash appeared, which involved her chest, neck, abdomen and groin and lasted five days. The rash was described by her mother as like a 'heat rash'. Her general practitioner described an exudative pharyngitis and cervical lymphadenitis, however did not observe other characteristics which can occur with scarlet fever such as the white/red strawberry tongue, circumoral pallor or 'Pastia's lines'. She was started on oral penicillin 24 hours after the onset of the illness.

The other three 'confirmed' cases of scarlet fever initially reported to the Public Health Unit had had similar clinical syndromes to the index case, with sudden onset of fever and sore throat followed by a rash. The GP could describe the characteristic white/red tongue for two children (who were siblings). None of the cases had desquamation after the rash. All were treated promptly by antibiotics, with the exception of one case who was treated in convalescence (after the result of the throat swab was obtained).

At the time of the notification the centre director was aware of 12 children who had been ill over the previous three weeks. All the doctors who had seen these children were contacted and interviewed about the clinical diagnoses, investigations and treatment. The mothers of these children were interviewed using a structured questionnaire either by telephone or face-to-face. The questionnaire was also distributed to the parents of the other children.

Throat swabs were taken from all the staff and the four children who had had a clinical illness consistent with scarlet fever (fever, sore throat, followed by rash).

Streptococcal antibody screens were taken from three of the children with an illness consistent with scarlet fever.

\section{CASE DEFINITIONS}

The following case definitions - for illnesses occurring in the day care centre children between June 1 and June 25 inclusive - have been adopted:

Case definition 1. Illness characterised by fever and sore throat followed by a rash. (Illness consistent with scarlet fever.)
Case definition 2. Illness characterised by a sore throat and fever. (Illness consistent with pharyngitis, bacterial and viral.) (Therefore case definition 2 includes case definition 1.)

Case definition 3. Illness characterised by one or more of the following: fever, sore throat, runny nose. (Illness consistent with an upper respiratory tract infection.) (Therefore case definition 3 includes case definitions 1 and 2.)

\section{PREDOMINANT SYMPTOMS}

Questionnaires for 48 out of the 72 children attending the centre (66.6 per cent) were finally returned. The rate of particular symptoms in these 48 children for whom information is available is shown in Table 3 :

\begin{tabular}{|c|c|c|c|}
\hline \multicolumn{4}{|l|}{ TABLE 3} \\
\hline Symptom & $\begin{array}{l}\text { Rate per } \\
100 \text { in } \\
\text { Group } 1\end{array}$ & $\begin{array}{l}\text { Rate per } \\
100 \text { in } \\
\text { Group } 2\end{array}$ & \begin{tabular}{|c|} 
Rate per \\
100 in \\
both groups \\
combined
\end{tabular} \\
\hline Rhinorrhoea & 56.7 & 38.9 & 50.0 \\
\hline Sore ears & 10.0 & 0.0 & 6.3 \\
\hline Sore throat & 33.3 & 11.1 & 25.0 \\
\hline Vomiting & 23.3 & 5.5 & 16.7 \\
\hline Rash & 20.0 & 0.0 & 12.5 \\
\hline Fever & 40.0 & 16.7 & 31.3 \\
\hline Headache & 13.3 & 0.0 & 8.3 \\
\hline Cough & 16.7 & 0.0 & 10.4 \\
\hline Abdominal pain & 10.0 & 0.0 & 6.3 \\
\hline
\end{tabular}

The most common symptoms in the 48 children about whom questionnaires were completed were: rhinorrhoea (50 per cent), fever (31.3 per cent) and sore throat ( 25 per cent). All symptoms were more common in group 1 children.

\section{CASE RATES}

The day care centre director was unaware of any other children - for whom questionnaires have not been returned - being ill since June 1 . (For the purposes of this discussion we will assume none of these other children became ill. This is a reasonable assumption for case definition 1 and possibly case definition 2 . The case rates for those with case definition $3-$ and to a lesser extent case definition $2-$ can only be underestimates.)

Out of 72 children who attend the day care centre, four (5.6 per cent) fulfilled the case definition of a clinical illness consistent with scarlet fever. All these children attended group 1; four of 45 (8.9 per cent) in group 1 therefore fulfilled case definition 1 .

An additional 11.1 per cent (eight of 72) of the centre's children had had a clincial illness consistent with pharyngitis, and seven ( 87.5 per cent) had received antibiotics without throat swabs.

Therefore there were in total 16.7 per cent (12 of 72) fulfilling case definition 2 . Of group 1 children, 22.2 per cent (10 of 45) fulfilled case definition 2 . Children in group 1 were three times as likely as children in group 2 to develop this illness, however this association was not significant at the 5 per cent level $(R R=3.0 ; 95$ per cent $\mathrm{CI}=0.71$ to $12.68 ; \mathrm{P}=0.10$ )

An additional 26.4 per cent (19 of 72) of the children had an illness consistent with an upper respiratory tract illness but without a sore throat. This was clearly the most common type of illness over this period. 
The frequency distribution over time of these various cases is illustrated in Figure 6. For many of those children who had had an illness consistent with an upper respiratory tract infection but who did not fulfil case definitions 1 or 2 , the parents did not complete the question referring to the onset of the symptom(s). The 'epidemic curve' therefore does not include these cases. (In addition, of the 11 staff members, 10 (90.9 per cent) had had a clinical illness consistent with an upper respiratory tract illness over this period.)

\section{LABORATORY RESULTS}

\section{Throat swabs}

Throat swabs were taken from the four children who had had a clinical illness consistent with scarlet fever, and all 11 staff on June 20. Group A beta-haemolytic streptococcus was isolated from two of these 15 throat swabs (13.3 per cent). These two positive swabs were from the two children who had not taken antibiotics beforehand.

Of the additional children fulfilling case definition 2 , the only swab which was taken - from the only one of these children who had not had antibiotics beforehand - was negative.

Of the additional 60 children who attended the centre, and who were advised to have throat swabs by their GP, the director of the day care centre estimated that 45 did attend their GP within the next two weeks. Only three of these children were found to be asymptomatic carriers of group A beta-haemolytic streptococcus.

\section{Streptococcal antibody screen}

On June 25, streptococcal antibody screens were taken from three of the children with an illness consistent with scarlet fever, including the two who had taken antibiotics before throat culture, and one who had had a positive throat swab.

Results of the three screens were all negative. (The blood tests were taken 23 days after the onset of illness for the index case, and 10 and eight days after the onset of illness of the other two cases.)

\section{PUBLIC HEALTH ACTION}

A letter was sent to the parents of the day care centre children on June 21 explaining that we had to presume there had been a minor outbreak of scarlet fever and a bacterial throat infection and that the recommendation was that the children have throat swabs taken by their GPs and if positive that they be treated with penicillin. An accompanying letter for the GP was enclosed.

\section{DisCUSSION AND CONCLUSIONS}

From a clinical viewpoint there are four reasons for diagnosing and treating group A streptococcal infections/ pharyngitis: the illness is shortened with early treatment; the organism is eradicated from the pharynx and cannot be spread to other individuals; suppurative complications can probably be prevented; and rheumatic fever and possibly acute glomerulonephritis are prevented.

From a public health perspective, it has been recommended that carriers be searched for and treated in 'welldocumented' outbreaks of streptococcal infection and that in outbreaks where individuals have close contact 'it may be necessary to administer penicillin to terminate spread'.

However, whether an outbreak of scarlet fever and/or streptococcal infection occurred in the day care centre is still unclear. Streptococcal infections are notoriously difficult to diagnose - in particular to differentiate from a number of upper respiratory tract viral illnesses - even with the benefit of adequate microbiology and serology 3 . In any case, serology is unlikely to be helpful in determining early whether a 'well-documented' outbreak is occurring as convalscent sera are usually necessary. In addition,

\section{FIGURE 6}

FREQUENCY DISTRIBUTION OVER TIME OF ILLNESS IN A DAY CARE CENTRE: JUNE 1991

5 NO OF CASES

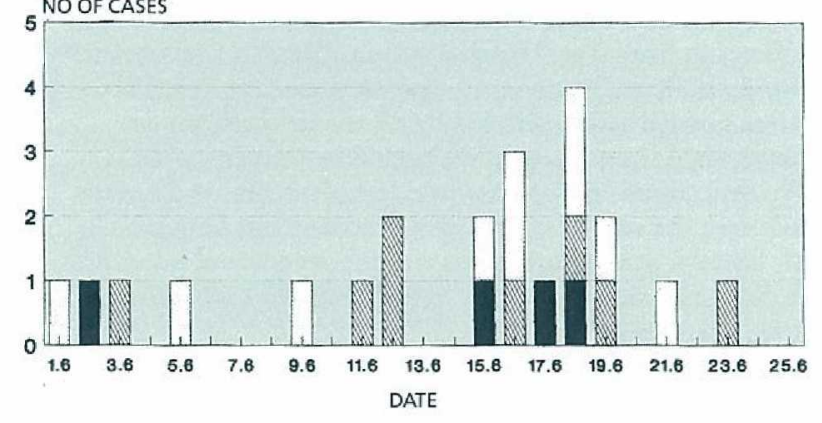

Case definition $1 \quad \square$ Case definition $2 \quad \square$ Case definition 3 For case definition see report

the utility of microbiological examinations is clearly diminished when suspected cases have already begun antibiotic therapy, which is often the case.

The symptomatic case definitions which were adopted in this investigation are clearly very broad, being consistent with diagnoses of scarlet fever and streptococcal pharyngitis; however the criteria used for these case definitions are far from diagnostic.

Prevalence rates of group A streptococci as normal asymptomatic inhabitants of the nasopharynx can vary from 15-20 per cent $t^{4}$. The two positive throat swabs from the two children with the clinical syndrome consistent with scarlet fever - who had not been started on antibiotics could easily have occurred by chance. Similarly, the three positive swabs from asymptomatic children lies within the bounds of what would be expected in a normal population.

Prompt treatment by antibiotics is known to inhibit the antibody response 4 . In addition, the degree of inhibition appears to be related to the successful elimination of the organism by therapy ${ }^{4}$; all three had negative throat swabs after antibiotics. Therefore the negativity of these three antibody tests is also an inconclusive result.

Most ill children over this period had a respiratory tract illness but without the sore throat and fever (and rash). The two possibilities remain that:

- All or almost all the 'outbreak' cases had another illness, most likely viral, which was difficult to distinguish clinically from both bacterial pharyngitis and scarlet fever; or

- There was indeed a small 'outbreak' of scarlet fever/group A beta-haemolytic streptococcus, superimposed on an 'outbreak' of a viral illness.

Although more extensive microbiology and/or serology may have provided firmer answers, this was not indicated given the nature, severity and prompt resolution of the 'outbreak', as a result of - or despite - the public health measures taken.

\section{Victoria Westley-Wise, Public Health Officer} Illawarra Public Health Unit

1. Denny FW. Effect of treatment on streptococcal pharyngitis: is the issue really settled? Paediatric Infectious Disease 1985; 4:352-4. 2. Benenson S. (ed). Control of Communicable Disease in Man. 15th ed American Public Health Association, 1990.

3. Kaplan EI, Top FH, et al. Diagnosis of streptococcal pharyngitis: differentiation of active infection from the carrier state in the symptomatic child. Journal of Infectious Diseases 1971; 123:5. 4. Denny FW, Perry WD. Type specific streptococcal antibody. Journal of Clinical Investigation 1957; 36. 